\title{
Optimization of control program for CNC machines with regarding dynamic stability of cutting process and optimum modes of treatment in conditions of digital production
}

\author{
Yuri G. Kabaldin*, Dmitrii A. Shatagin, and Pavel V. Kolchin \\ Nizhny Novgorod State Technical University n.a. R.E. Alekseev, 603950 Nizhny Novgorod, Russia
}

\begin{abstract}
The method for optimizing control programs for CNC machines based on artificial intelligence approaches, in particular, the apparatus of artificial neural networks, is outlined. A neural network model of the dynamic stability of the cutting process is proposed, which makes it possible to simulate the dynamics of the cutting process using the CAM system.
\end{abstract}

\section{Introduction}

At present, there is no single basic control platform [1], on the basis of which a new generation of control systems for technological equipment for digital production will be developed. Such platforms should have large computing resources and a high processing speed for processing a large database. This will significantly reduce costs both for the development and operation of technological equipment, and ensure its entry into a single information space of the whole enterprise. The lack of a basic platform for managing technological equipment also constrains the development of new high-performance methods for manufacturing parts (high-speed processing, additive technologies, hybrid processing technologies, etc.) and, in general, the efficiency of digital production. The creation of a unified equipment management platform will solve another important task: technological security of the country as a whole. most of the existing equipment, including those at defense enterprises, are equipped with imported equipment and control systems.

Therefore, the formation of a new approach to the management of digital production equipment and the development of a basic unified platform, the creation on its basis of both new and improvement of existing control systems for process equipment, is an urgent task for digital production.

Such a single platform can be open systems of CNC machines [2-8] with built-in modules of high-performance computing and in-depth training of artificial intelligence (AI) using nVidia CUDA technologies, which provides the possibility of scaling based on graphics processors (GPUs) and communication modules with industrial Internet, i.e. with the possibilities of using cloud technologies for processing large data both on enterprise

* Corresponding author: uru.40@mail.ru 
servers (local networks) and on servers of providers. All this creates the basis for intellectual management of a wide range of technological equipment equipped with CNC systems with advanced capabilities, in particular, in high-speed processing equipment, additive technologies, etc., and also integration of diagnostic subsystems, for example, tool wear, into it. At the same time, special attention should be paid to the development of diagnostic systems for the dynamic state of machining equipment.

\section{Methods}

In modern computerized production, as a rule, all the necessary information about a given range of manufactured parts can be extracted from the CAD database or from a corporate PDM system (project management system). In accordance with this information, the task is to determine the optimization of the control program (PM) and the modes of processing the parts.

The fact is that the quality and productivity of machining parts will be affected both by the dynamic state of machine tools and by inefficient cutting modes that can cause chaotic operation of equipment and reduce processing capacity [3]. In this connection, when working with CAM-systems, it is necessary to optimize the process of forming the control program of a $\mathrm{CNC}$ machine based on non-linear dynamics and artificial neural networks (INS). Algorithms of nonlinear dynamics allow us to evaluate the randomness of the motion of the machine's executive bodies and optimize the trajectory of the tool movement, and the INS to optimize the machining regimes of parts [2-4].

Information on the relative vibrations of the tool and the workpiece can be obtained only as a result of indirect measurements. The main task of analyzing such data is to determine the qualitative and quantitative characteristics of the detected signals, which allow us to judge the true state of the dynamic processes occurring in the cutting zone.

The presence of many degrees of freedom of the elastic system of a metal-cutting machine $[9,10]$ leads to the fact that the oscillations of the tool relative to the workpiece represent the result of the addition of several interconnected elementary (translational or torsional) oscillations. Therefore, the trajectory of the relative motion of the tool and workpiece, formed as a result of the addition of oscillations, has the form of a closed curve, theoretically the shape of the ellipse (Fig. 1). This ellipse is commonly called the "ellipse of displacements" [2].

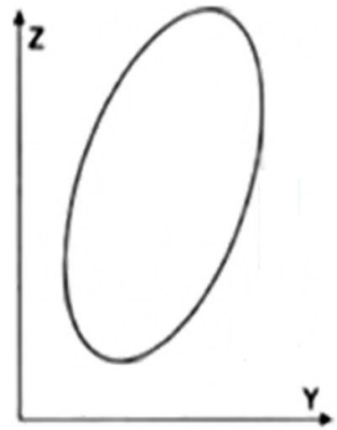

a)

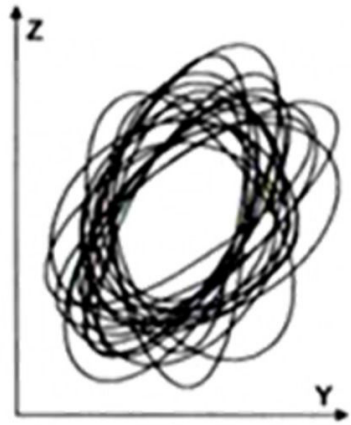

b)

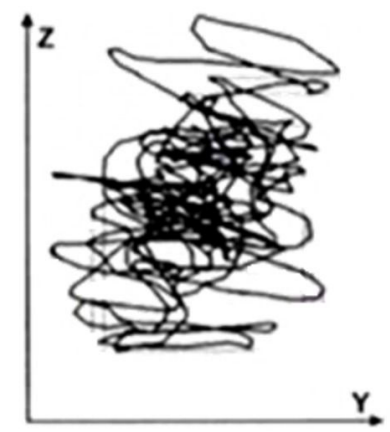

c)

Fig.1. Trajectories of the relative motion of the tool and workpiece during turning: a) theoretical (displacement ellipse); b) obtained as a result of refined computer modeling taking into account perturbations; c) fixed by means of a system of polarized vibration sensors mounted on the tool holder. 
In reality, due to various perturbations, the trajectory of the tool tip movement [2-4] has a chaotic appearance (Fig. 1b, c). That is, the theoretical "ellipse of displacements" for a given steady-state cutting mode is a kind of attractor-an attractive set of points in the phase space of the relative position of the tool and the part.

To fix the real trajectory of motion of the tool tip, with sufficient accuracy for effective diagnostics, it is possible to use only special precision equipment, for example, using a laser interferometer. Naturally, the use of such complex and expensive equipment as part of control systems for metal-cutting machines is not economically feasible because of too high adjustment and maintenance costs. Therefore, as a rule, cheaper but less accurate instruments are used to measure oscillations during cutting. The widest application in this area has been the use of vibration sensors and acoustic emission sensors [2-4].

Polarized (having sensitivity along one axis) vibrating sensors are attached to the elements of the elastic system of the machine in such a way that together they form an orthogonal system. This method of positioning allows one-dimensional signals of sensors fixing one of the projections of oscillations to form a three-dimensional spatial picture.

However, due to wave phenomena in the elastic system of the machine, the vibration sensors, in addition to the useful signal, fix a sufficiently large number of side oscillations caused by reflections of the waves. There is a deformation from a variety of joints and cross sections of the elements of the machine's structures located near the place where the sensors are fastened. As a result of this, the trajectory of motion of the tool tip [2-4] reconstructed from the results of vibration measurements is highly distorted (Fig. 1c).

To study the relative oscillations of the tool and the workpiece during cutting, a neural network dynamic model of the oscillating system "tool-detail" was developed. The application of this model allows the dynamic diagnostics of the relative movements of the tool and the workpiece, using inexpensive vibro-registering sensors and current sensors, to be carried out directly in the process of cutting with an accuracy comparable to the results of laser interferometry.

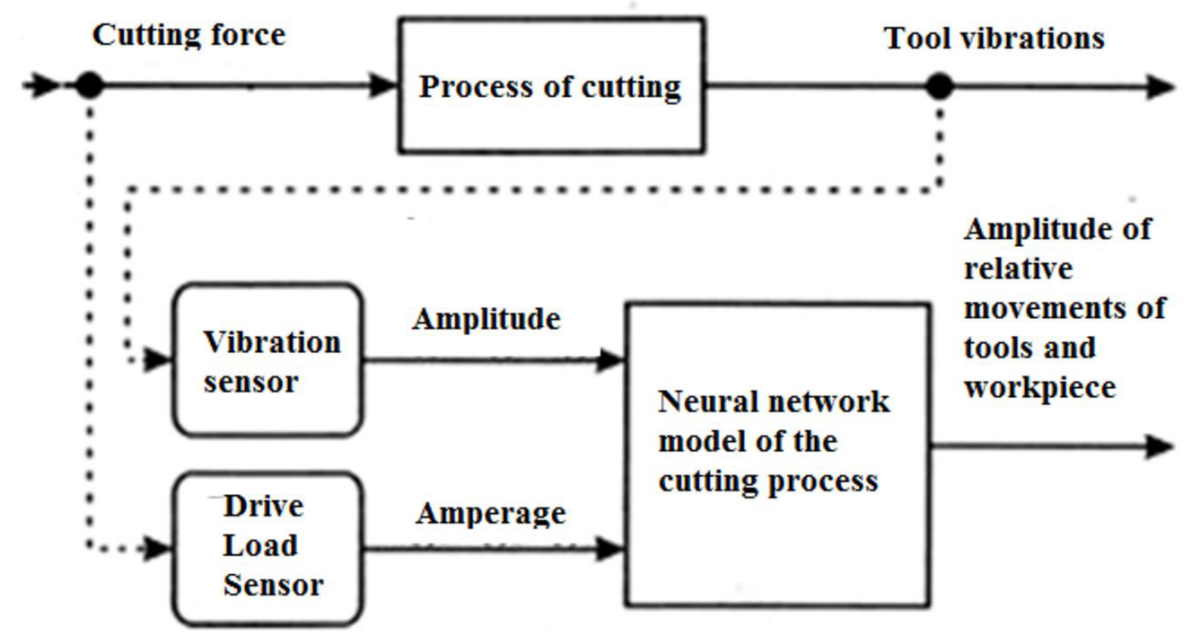

Fig. 2. Diagram of the diagnostics of the relative movements of the cutting tool and the workpiece using the neural network dynamic model of the oscillating system "tool-detail"

The scheme of the diagnostic process using the model is shown in Fig. The amplitude of the signals of the orthogonal system of polarized vibration sensors $S(t), S y(t), S z(t)$ 
located on the elements of the elastic system of the machine, as well as the effective values of the current in the windings of the main drive motors Iz ( $t$ ), feeders Ix ( $t$ ) and Iy ( $t$ ).

As the output data, the amplitude values of the projections of the relative movements of the workpiece and the tool Ax (t), Ay (t), Az (t) are taken. The current values of the currents characterize the quasistatic, slowly varying components of the cutting forces $\mathrm{P}$, and the signals of the vibration sensors allow us to estimate the dynamics of the change in these forces.

The dynamic model of the "tool-detail" oscillatory system is formed on the basis of a two-layer neural network of backward propagation. The objective of the network is to approximate the trajectory parameters of the tool tip (the dimensions of the displacement ellipse, the angle of its inclination with respect to the coordinate system) and the estimated values of the amplitude of the relative movements of the workpiece and the tool in the projections.

Figure 3 shows the structure of the neural network dynamic model of the oscillating system "tool-detail"

The first, the input layer of the network (Fig. 3) contains 16 neurons, the second, the output layer consists of 3 neurons. As an activation function for the neurons of both layers, an exponential sigmoid is used. The network has 240 inputs to which the actual values of the vector of the physically measured quantities are fed $\psi(t)=S x(t), S y(t), S z(t), I z(t), I x$ $(\mathrm{t})$, Iy ( $\mathrm{t})$, a also the history of this vector for 39 previous time readings $\psi(\mathrm{t}-1), \psi(\mathrm{t}-39)$, formed by means of shift registers $(\mathrm{R} 1+\mathrm{R} 39$ in the figure). The three outputs of the network are the outputs of the model.

The input vector $\mathrm{P}(\mathrm{t})$ of the network is formed in the time domain discretely, on the basis of synchronous measurements of the instantaneous values of the vibration sensor signals Sx, Sy, Sz and the current strength Ix, Iy, Iz in the windings of the drive motors. The measurements are carried out in real time with a sampling frequency of 10,000 Hz. Regularly, with a frequency of $10 \mathrm{~Hz}$, based on the time series captured by the instantaneous values, the vibration amplitudes $\mathrm{Sx}(\mathrm{t}), \mathrm{Sy}(\mathrm{t}), \mathrm{Sz}(\mathrm{t})$ and the current values of the currents Iz $(t)$, Ix $(t)$, Iy $(t)$, that is, the next time count of the vector $\psi(t)$ is given.

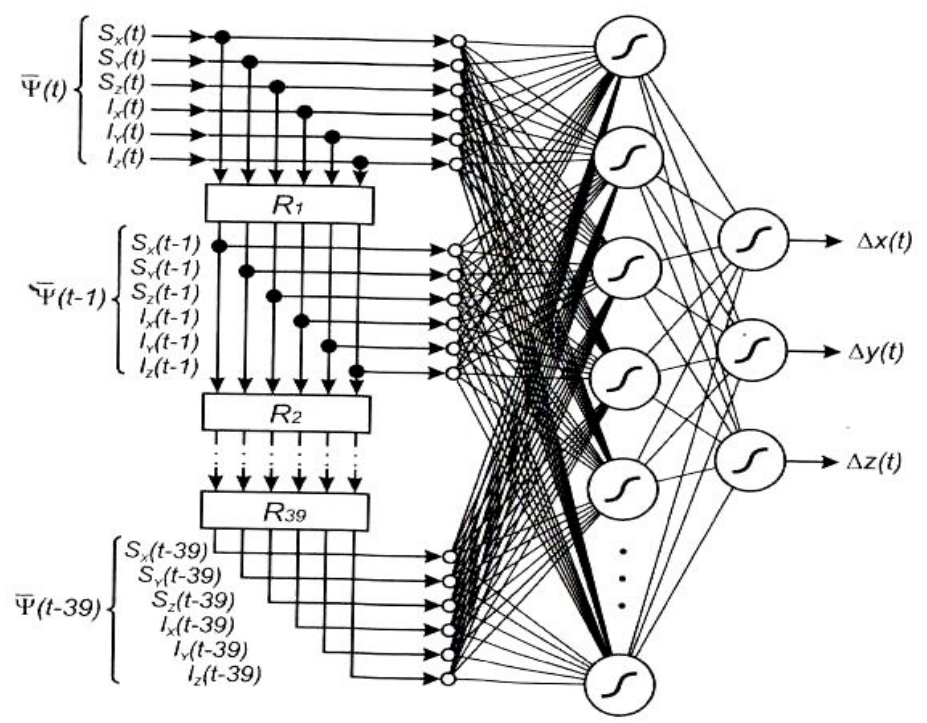

Fig. 3. Structure of the neural network dynamic model of the oscillating system "tool-detail". 
According to calculations, the reaction time of the model is 0.1 seconds, the storage capacity for the history of development of oscillatory processes in the "tool-detail" system (the maximum duration of the considered transient phenomena) is 4 seconds.

Simulation of dynamic parameters of the oscillating system "tool-detail" is carried out by training the network. The training sample was formed on the basis of a comparison of the values of the input parameters for various variations of the cutting regime and the relative displacement values of the workpiece and the cutting tool Ax (t), Ay (t), Az (t) determined by a laser interferometer.

The network is trained using the algorithm for back propagation of the error [2-4]. To avoid the effect of "retraining" the final step in the formation of the network is the procedure of contrasting the synaptic scales.

After training, the neural network is able to identify the dynamic state of the elastic system of the machine (to distinguish between stable cutting and unstable, to predict the parameters of the trajectory of the tip of the tool) and to diagnose the amplitude values of the oscillations of the cutting tool relative to the workpiece in the projections on the axis of the machine coordinates.

\section{Results}

The hardware-based neural network model can be implemented on the basis of a small number of microelectronic components, most of which are widely used in automated process control systems. To dynamically register the instantaneous values of Sx, Sy, Sz, Ix, Iy and Iz, the hardware model should include an analog switch and an analog-to-digital converter. Control of the input devices of the model, the vibration amplitudes Sx (t), Sy ( $t)$, $\mathrm{Sz}(\mathrm{t})$ and the current values $\mathrm{Iz}(\mathrm{t})$, Ix $(\mathrm{t})$, Iy $(\mathrm{t})$ should be assigned to the signal processor, and the entire computational load by Neural network processing will be performed by a specialized neural network processor (neurochip) containing in its memory registers a neural network dynamic model of the instrument-detail oscillation system obtained at the training stage. As the final driver of the output parameters of the Ax (t), Ay (t), Az (t) models, a digital-to-analog converter can be used.

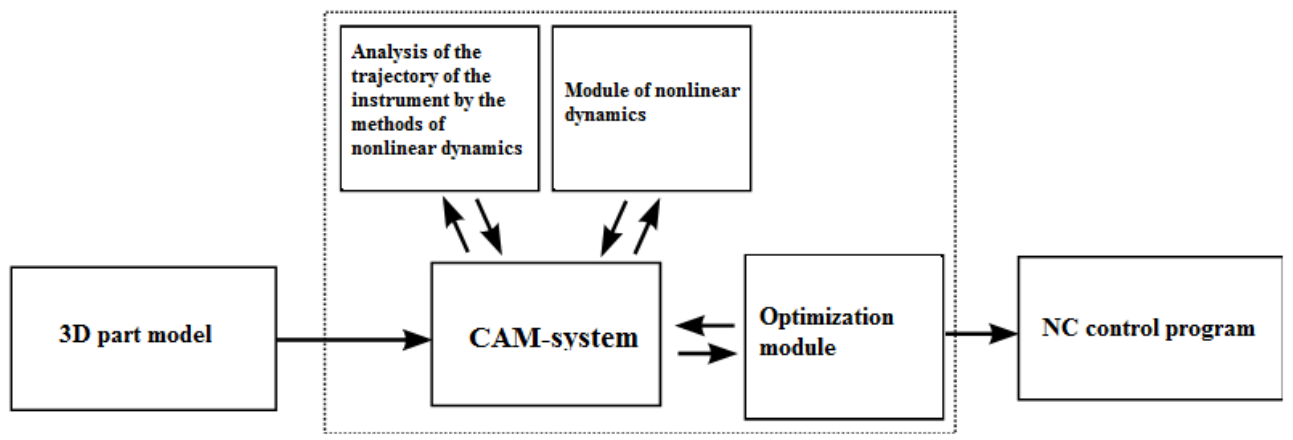

Fig. 4. The process of forming and optimizing the control CNC program taking into account the dynamic characteristics of the machine.

Figure 4 shows the scheme for the formation and optimization of UE.

It follows from Fig. 4 that, for optimization, the UE should be supplemented with the following procedures:

- an estimation of chaos of UE by methods of nonlinear dynamics;

- Neural network optimization of the tool path;

-network optimization of processing modes. 
The algorithm of interaction of these program modules with the core of the CAMsystem can be represented in the form of a block diagram, shown in Fig.

At large enterprises, there is a database (DB) that provides automation of both tool selection and cutting modes directly from the CAM system and transferring the parameters of the recognized structural elements from the 3D model of the workpiece to the cloud, and back receives a list of tools and recommended values for processing modes . Moreover, it should be noted that the imported instruments serve not only for the subsequent calculation of trajectories and verification, but also are provided with catalog numbers, which greatly facilitates the work of the technologist responsible for selecting and acquiring the tool necessary to carry out the current production assignment.

This approach provides access to extensive production data in the cloud, primarily data on the cutting tool and tooling, which require large computational capabilities for analysis. In this case, a great benefit for users is that the responsibility for filling the actual data of the cloud falls on the consumer, not the developer of the CAM-system or third-party companies engaged in filling the database. This largely applies to defense enterprises. To implement such a concept, two difficult tasks must be solved: first, to negotiate with manufacturers of equipment about the regular work to fill the cloud with data about their products, and, secondly, to create their own set of applications for accessing the cloud from various operating systems and thus to provide access to the cloud even with the help of a smartphone in this production, i.e. by implementing the "Internet of things".

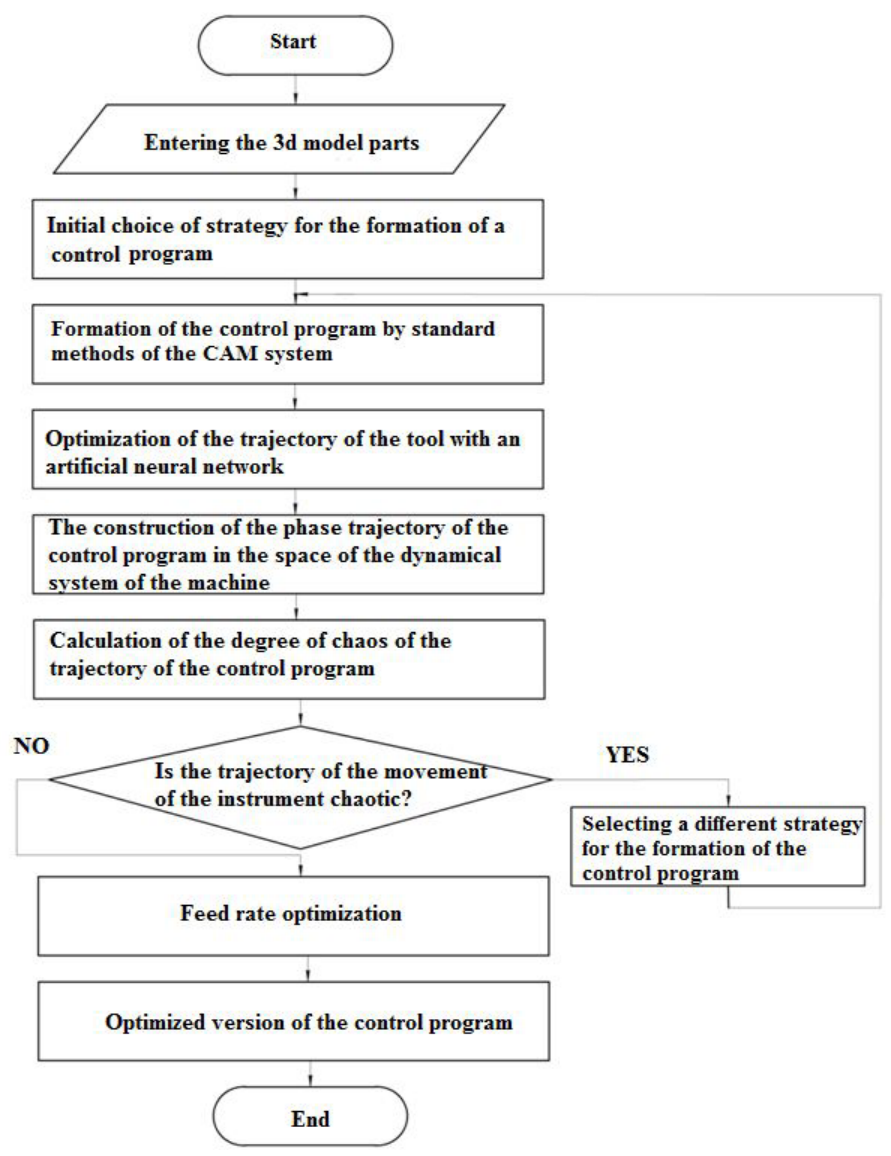

Fig. 5. Algorithm for the formation and optimization of the CNC control program with the use of ANN and nonlinear dynamics. 
As a basis for implementing neural networks in the optimization of UE, a hardware neuroxybernetic device can be used, for example, the domestic neuroprocessor NeuroMatrix (NM6403). Due to its versatility, the neuroprocessor can be used as a base element of neuro-accelerator boards for high-performance computers, including for computer centers oriented to CAD applications.

\section{Conclusion}

Based on the optimization approach described above, the program module "NeuroModule UG" for the NX CAM system was created [2] The module was developed using the UG Open API mechanism and allows to supplement the full-time capabilities of the NX CAM system with the procedure of automated tool path formation for high-speed milling of a wide class parts of the aircraft industry, such as a frame, spar, rib, containing a large number of technological elements such as "pocket".

The module independently determines the quantity and geometry of the "pockets" and forms for each of them several trajectories of the cutting tool movement. Then the best trajectories are selected from the positions of high-speed machining, which allows to fully reveal the advantages of high-speed milling for this form of "pocket".

The application of dynamic models of machining processes, built on the basis of neural networks for controlling metal cutting machines, makes it possible to control the dynamic stability of the cutting process, while at the same time improving the effect of processing quality, dimensional accuracy, and also improving tool life.

\section{References}

1. V.L. Sosonkin, G.M Martinov, Devices and control systems, 8, 18 (1996)

2. Y.G. Kabaldin, S.V. Bilenko, S.V. Seryj, Bulletin of mechanical engineering, 1, 50 (2003)

3. Yu.G. Kabaldin, S.V. Bilenko, S.V. Seryi, Control of dynamic processes in technological systems of machining based on artificial intelligence (Komsomolsk-onAmur state. tech. un-t, Komsomolsk-on-Amur, 2003)

4. Y. G. Kabaldin, S. V. Bilenko, and S.V. Seryi, Russian Engineering Research, 3, 25 (2003)

5. Y. G. Kabaldin, I.L. Laptev, D.A. Shatagin, S.V. Seryj, Bulletin of Machine Building, 8, 80 (2014)

6. Yu.G. Kabaldin, I.L. Laptev, D.A. Shatagin, V.O. Zotov, S.V. Seryi, Machine building: a network electronic scientific journal, 2, 47 (2014)

7. Yu.G. Kabaldin, I.L. Laptev, D.A. Shatagin, Yu.V. Vytnov, S.V. Golubev, Proceedings of the NSTU, 5, 114 (2013)

8. Yu.G. Kabaldin, D.A. Shatagin, I.L. Laptev, D.A. Sidorenkov, Izvestiya Vysshikh Uchebnykh Zavedenii, 10, 49 (2016)

9. V.A. Kudinov, Dynamics of machine tools (Mechanical Engineering, Moscow, 1967)

10. M.L. Orlikov, Dynamics of machine tools (High School, Moscow, 1989) 